\title{
Down Syndrome and Quality of Life: A Case Report
}

Hilary L. Schroeder, ${ }^{1}$ Marianinha Joanes, ${ }^{2}$ Raghu Maramraj, ${ }^{3}$ Andre Small.'

\begin{abstract}
Background: Quality of life is considered a crucial component to the well-being of patients with Down syndrome. The strength of quality care through stable social and psychological interactions has built a framework for a positive well-being for patients with Down syndrome, improving their quality of life. Case: A 55-year-old African American female with a history of Down syndrome, congenital heart disease, and newly-diagnosed early onset Alzheimer's disease presented with an arm contusion resulting from regular caretaking. The patient's history was reviewed, and the complexity of her condition was discovered. While a subset of Down syndrome patients have cardiac complications and others have early-onset Alzheimer's, our patient had both. We believe this complicated her condition. After the diagnosis of Alzheimer's was made, the caregivers noticed a significant decline in her ability to communicate and continue day-to-day activities. Despite the decline in functions, a positive mood was apparent. Conclusion: Multiple medical interventions, along with strong family support, positively contributed to the patient's quality of life. Therapies targeting cognition could result in the maintenance of quality of life and, ultimately, lower health care costs.
\end{abstract}

Keywords: Down Syndrome; Alzheimer Disease, Early Onset; Heart Defects, Congenital; Quality of Life (Source: MeSH, NLM).

\section{Introduction}

About the Author: Dr. Hilary Schroeder graduated recently from the American University of Antigua in St. John's, Antigua, West Indies. She is currently completing her first year of Family Medicine residency at the Institute for Family Health in Kingston, New York.

Submission: Oct 5, 2014

Acceptance: Apr 9, 2015

Publication: Aug 31, 2015

Process: Peer-reviewed

\section{The Case}

Quality of life is considered a crucial component to the well-being of patients with Down syndrome. Down syndrome treatment should include quality of life improvement measures because although an increase in longevity may not be observed, maintained communication and social interactions can.' The strength of quality care through stable social and psychological interactions has built a framework for a positive well-being for people living with disabilities. ${ }^{2}$ Quality of life is increasingly being recognized as a measuring tool to assess the progression of disease. ${ }^{3}$ Within the definition, quality of life encompasses the overall environment and includes the families and friends of Down syndrome patients who provide the highest degree of care. Through lifestyle modifications, medical interventions and strong support networks, patients with Down syndrome can increase their life expectancy. ${ }^{4}$ In this paper, we demonstrate how quality of life is important to Down syndrome patients and why quality of life is an important measurement in treating these patients.

Our patient was a 55-year-old African-American female brought into the Emergency Room by her father (primary caregiver who gave informed consent for this report) in September 2012 with concerns about a large contusion on her arm from routine caregiving. Her past medical history included Down syndrome (1961), mitral valve dysfunction (family could not recall time of diagnosis), and cerebrovascular accident (2010). Patient had no known allergies. Medications included: miconazole (Monistat), warfarin (Coumadin), pantoprazole (Protonix), zinc oxide, phenytoin (Dilantin), protamine sulfate, digoxin (Digox), and acetaminophen (Tylenol). Surgical history included two pros-

Key Points:
- Quality of life can be difficult to assess, therefore regular interaction
with patients is necessary.
- Comorbidities play a significant role in the lives of Down syndrome
patients and can affect their overall quality of life.
- Allowing Down syndrome patients to be interactive and participate in
daily activities is important for their well-being.
- Positive environments with clear and constant communication play
a crucial role in establishing improved quality of life among Down
syndrome patients.
- Quality of life should be taken into consideration when assessing the
effectiveness of treatment.

thetic valve placements (family could not recall time of surgery, but associated it with mitral valve dysfunction diagnosis), Medtronic AT5000 series cardiac pacemaker implantation (2005), supraventricular ablation (2009), and G-tube placement (2012).

The patient's father was alive and well at 79 years old, while her mother was 24 years old when birthing her and died at 43 years old from metastatic breast cancer. The patient's paternal grandmother had Alzheimer's disease. The patient lived with her father and sister (another caregiver giving informed consent). She was born overseas, had never been employed, but did attend sheltered workshops. She thoroughly enjoyed regularly attending church. Her family denied any alcohol, tobacco, or illicit drug use.

During a home visit in October 2012, we observed our patient, who was bed-bound and unable to communicate her needs effectively, receive exceptional family care. The patient had 24-hour care by her father and daily visits by her sister and a nurse supplied by the hospital. They constantly integrated her into conversations and daily tasks, thereby improving her

American University of Antigua College of Medicine, US.

${ }^{2}$ International American University College of Medicine, Canada

${ }^{3}$ University of Sint Eustatius School of Medicine, US.

Correspondence:

Hilary L. Schroeder

Address: American University of Antigua, Saint George, Antigua and Barbuda.

Email: hilary0833@yahoo.com 
independence; this was beneficial through her noticeable positive mood. During our time with her, we observed her gain the skills to feed herself and very minimally converse. Her father and sister were vigilant about tending to her and ensuring that she try and at least maintain her new skills. They ensured prompt organization of appointments for her cardiac surgeries and follow-up appointments at the hospital and with our team.

\section{Discussion}

\section{Family Care and Deinstitutionalization}

Quality of life can be deemed as subjective; however, it is increasingly being recognized as an asset in the monitoring of disease progression. ${ }^{5}$ Strong family support, solid communication, and long-lasting friendships have all contributed to increasing the quality of care for patients with Down syndrome. Continuous support and attentiveness by the patient's father and sister allowed the patient to be interactive and learn new tasks. They carefully monitored her, yet allowed her to practice independence by interacting with the people in her community and attending church. This added to her quality of life. Time spent with her family provided us insights on her ability to properly communicate with caregivers and their ability to appropriately interpret her requests. A positive environment empowers Down syndrome patients to continue to learn and teach themselves small tasks in their daily lives. ${ }^{6}$ External factors such as socioeconomic factors, strong community support, and proper daily resources all influence the overall well-being of patients with Down syndrome and increase their ability to function to their maximum capacity.? Quality of life involves family, environmental and emotional support.

\section{Strengths and Limitations to Our Approach}

For six months, our group made multiple home visits and interacted with the caregivers to better understand her changing medical status. Over time, it was clear her condition was becoming more complex. As her cognition declined, we relied more on the caregiver's interpretation of her feelings. Throughout the six-month follow-up period, we maintained constant communication, tracked patient's progression, and witnessed the importance of a positive environment and family support. Historical data gathering was limited by incomplete hospital charts, lack of verbal communication by the patient, and difficulties with data recall by the family.

\section{Conclusion}

It is our belief that a combination of advanced medical interventions and support greatly improve the quality of life in Down syndrome patients. Treatment of comorbidities, therapies targeting cognition, and added support for patients with Down syndrome help increase their involvement in daily activities, social bonding, and quality of life. 


\section{Case Report}

\section{References}

1. Goodman MJ, Brixner DI. New therapies for treating Down syndrome require quality of life measurements. Am J Med Genet A. 2013 Apr;161A(4):639-41. 2. Felce D, Perry J. Quality of life: the scope of the term and its breadth of measurement. In: Brown RI, editor. Quality of life for people with disabilities: models, research and practice. Cheltenham (UK): Stanley Thornes; 1997. p. 56-71.

3. Raphael D. Quality of life of persons with developmental disabilities: five issues concerning its nature and measurement. J Dev Disabil. 1997;5(2):44-66. 4. Glasson EJ, Sullivan SG, Hussain R, Petterson BA, Montgomery PD, Bittles
AH. The changing survival profile of people with Down's syndrome: implications for genetic counseling. Clin Genet. 2002 Nov;62(5):390-3.

5. Smith DS. Health care management of adults with Down syndrome. Am Fam Physician. 2001 Sep 15;64(6):1031-9.

6. Brown RI. Personal Reflections: quality of life research and Down syndrome. Int J Disabil Dev Educ. 1998;45(3):323-9.

7. Brown RI, Brown PM, Bayer MB. A quality of life model: new challenges arising from a six-year study. In: Coode $D$, editor. Quality of life for persons with disabilities: international perspectives and issues. Cambridge (MA): Brookline Books; 1994. p. 39-56.

\section{Acknowledgments}

None.

Conflict of Interest Statement a Funding

The author has no funding, financial relationships or conflicts of interest to disclose.

Author Contributions

Conception and design the work/idea, Collect data/obtaining results, Analysis and interpretation of data, Write the manuscript, Critical revision of the manuscript, Approval of the final version: HLS, MJ, RM, AS. Administrative or technical advice: HLS.

Cite as:

Schroeder HL, Joanes M, Maramraj R, Small A. Down Syndrome and Quality of Life: A Case Report. Int J Med Students. 2015 Apr-Aug;3(2):112-4. 